\title{
3D Virtual Spaces Supporting Engineering Learning Activities
}

\author{
D.M. Popovici, J.P. Gerval, F. Hamza-Lup, R. Querrec, M. Polceanu, N. Popovici, R. Zăgan
}

\author{
Dorin-Mircea Popovici, Mihai Polceanu, Norina Popovici, Remus Zăgan \\ OVIDIUS University of Constanta \\ Address: 124 B-dul Mamaia, 900527, Constanta, Romania \\ E-mail: dmpopovici@univ-ovidius.ro,polceanum@gmail.com,norinapopovici@yahoo.com, \\ rzagan@univ-ovidius.ro \\ Jean-Pierre Gerval \\ ISEN \\ 20, rue Cuirassé Bretagne \\ CS 42807 - 29228 Brest Cedex 2 - France \\ E-mail: jean-pierre.gerval@isen.fr \\ Felix G. Hamza-Lup \\ Armstrong Atlantic State University \\ Faculty of Computer Science \\ Address: 11935 Abercorn St., Savannah, GA \\ E-mail: felix@cs.armstrong.edu \\ Ronan Querrec \\ Ecole Nationale d'Ingénieurs de Brest \\ Laboratoire d'Informatique des Systèmes Complexes \\ Address: 25 rue Claude Chappe, F-29280 Plouzané, France \\ E-mail: querrec@enib.fr
}

\begin{abstract}
Virtual environments constitute the support platform for various teaching and learning activities. Instead of a local application for this purpose, this paper explores the effects of using distributed virtual reality environments in the educational process. The architecture of the presented system is based on the recently developed web-based technology called AJAX (Asynchronous Javascript And XML), implemented on a Linux operating system configured to run Apache with PHP and MySQL support; i.e., LAMP architecture, which contributes to the portability and ease of installation of the application. The platform is designed to support the integration of lesson modules such as the EngView environment which is discussed in more detail in this contribution. Pedagogical, technical, and implementation-related aspects are presented in conjunction with the virtual environment used in the engineering training curriculum. Statistical information resulted from the training shows a significant increase in task completion time when the virtual setup is used.

Keywords: Virtual environment, Learning, Teaching, Motivation, Virtual training.
\end{abstract}

Knowledge acquisition has shifted from an individual to a collective activity. There is a migration of the learning process from one individual to a group of individuals as knowledge becomes a collective activity enhanced by the phenomena of social interaction. The complexity of the information and the way we interact with it makes us active parts in the educational environment. Searching, discovering, and testing are the most frequent human activities in such situations. When an interpretive level of comprehension is reached, these activities are mature enough to trigger creational thinking, and constitute the beginning of the applied level of understanding. As complements to learning, virtual training gives constructive feedback to learners by providing them with a hands-on approach to the studied subject.

In the following sections, we emphasize the potential of distributed virtual environments to improve the learning process. To prove the point, we try to answer one question: "What do the $3 D$ virtual spaces bring into the learning processes in order to make them effective and evolutionary?" 


\section{Introduction}

A three-dimensional (3D) virtual space is a computer-generated space that is perceived by us via pure virtual reality (VR) technologies and/or mixed reality (MR) technologies [1]. This perception can only be obtained through placing the user in the space, from the user's interaction with the space. Furthermore, this space is not passive since the users interact with each other and/or with other virtual entities, by the means of virtual agents or avatars. Virtual objects are subjects in the users' direct or indirect interactions and may enhance collaboration between users. Users' multimodal communication is realized through exchanging typed or verbal messages, gestures, and facial expressions.

In other words, the virtual space must be constructed, first of all, considering the user's cognitive and empirical attributes. This means that when we create virtual space models, the base criterion should be the accuracy of the human representation of reality which may not necessarily correspond with reality. To this end, the human experience is first constructed by situating the user in the virtual context, then tested through the user's direct interaction with the environment, and later reconsidered, in a recursive process.

How efficient and effective is such experience? A possible answer may be given by evaluating the user experience in the frameworks proposed by Burdea [2] and Zelter [3]. We do not discuss the imagination aspect of the user experience but leave it private to the user. It may surprise the reader, but we are not trying to obtain an accurate sensorial rendering of the virtual space in order to immerse the user in the environment. Instead, by high quality immersion inside this virtual space we mean intense user interactivity with objects or other users within the virtual space.

The efficiency and the effectiveness of such experiences are considered acceptable if the user is able to apply the knowledge and skills obtained in the virtual space in similar real-space conditions; i.e., if both the gained knowledge and skills are reusable.

Applying such virtual spaces in learning and teaching activities provides the conditions for transforming the sometimes passive actors (i.e., students and teachers) into involved, very active actors [4]. With the support of new technologies, we hope to infuse them the sensation that they represent active parts of the learning/teaching process [5].

Our goal is to catalyze the creative state-of-mind and self-confidence at an individual level as premises of collaboration among individuals, with personal perspective as the basis for the learning communities. These communities provide the necessary conditions for transforming the users' interactions, expressed through direct communication and cooperation with other individuals, into long-term social interactions.

Many educational virtual environments such as "virtual theatre" and "virtual classroom" use various metaphors to facilitate the trainee in learning on an abstract (e.g., math, physics, electronics, and other $[6,7,8,9])$ or concrete (e.g., gesture or behavior in certain situations $[10,11])$ level. Few environments take into consideration the trainee's motivation to learn. Driven by this observation, our goal is to obtain a solution designed to serve as a motivational feedback to its users.

Virtual theatre or narrative-based metaphors have one major advantage as compared to other metaphors; i.e., they challenge and encourage the user to verbalize/render his/her experience in a situational context. Such an environment is highly evolutional since every actor comes with his/her own personal experience in a similar situation; this way knowledge is collectively and continuously modeled to better express the social point of view. The more we express the knowledge, the better the result becomes. Multimodal environments that combine haptic feedback with 3D visualization and sound rendering [12] prove to be very efficient learning tools, especially for understanding abstract concepts.

As students gradually gain confidence in the team they belong to, they become autonomous and willing to acquire new knowledge; thus, they change from being dependent on the team to being independent, and the relationships among individuals become dynamic and friendly. In particular, team-based environments are suitable for interdisciplinary teams. For example, the EngView system about which we discuss in this paper was developed by a mixed team of computer scientists, engineers, and managers, as 
well as a group of enthusiastic students. Engaging such a team, we have attained our main pedagogical objective that is to assure a rapid and successful integration in the professional context for our students. However, some difficulties rise due to factors such as the different levels of knowledge acquired by students during their studies, the student's level of interest in the information presented and the student's motivation to learn.

Learning speed varies from person to person. Often, theory is easier to grasp than to translate into practice. Or vice-versa, practical skills are quickly acquired, even without any basic understanding of the theory. Despite these difficulties, students need to achieve good theoretical and practical skills.

At the theoretical knowledge level, the widely used method of multiple choice examinations can be computer-graded or easily marked with a template. However this method does not provide any insight into the trainee's work methods and adaptability. A much better choice is a written examination. On the other hand, practical examinations are somewhat more probing; however, the trend is to have the candidate demonstrate his/her skills in a simple application where the results can be easily and uniformly graded [13].

Because paradigms such as VR and multimodal environments facilitate learning through the construction of concepts relying on the intuition that arises from direct user experience in the virtual environment [14], we decided to complement our teaching/learning process by using these technologies. We do not eliminate multiple choice examinations, but we consider that communication and interaction within a collaborative virtual environment may represent essential motivational dimensions to the trainee. Therefore, we consider interaction and communication as being the most important requirements of VR-based training technologies.

Another important aspect is the reduced accessibility of the real training setups for a group of trainees. By means of switching between training sessions in real environment and virtual replicas, the trainee is able to obtain the confirmation of his/her practical results obtained in the virtual environment. Thus, we do not eliminate traditional assessment, but we let the students exercise longer within a virtual setup, without any physical risks and at potentially lower costs. When students reach a certain level of "virtual expertise", they are allowed to prove this expertise in a real environment.

\section{EngView - a training tool for engineers}

To demonstrate the effectiveness of the educational concepts mentioned above, we implemented a training environment for engineers, called EngView [15], that is a supplementary tool in the training process in the domain of non-destructive testing (NDT), as detailed in the next section.

Because the presented training environment addresses mature users, motivation may not necessarily come from the environment itself, but from the user's desire to succeed in his/her integration within a professional context. In such a context, social interactions frequently appear in team setups and trigger individual development on both theoretical and practical levels. Due to frequent switching between experimentation and theory, it is not surprising that discovery, creation, and innovation are expected side-effects in engineering learning contexts.

\subsection{NDT principles}

The most used formats in the NDT training process are the A-scan, B-scan, and C-scan presentations. These provide different ways of visualizing and evaluating the inspected material region. For our purposes, we have chosen to visualize only the $\mathrm{C}$-scan method.

The high-frequency ultrasonic $\mathrm{C}$-scan presentation provides the planar view, depth location, and size of the defects inside the probe; this makes $\mathrm{C}$-scan a valuable tool to monitor the precise location of the defects between certain layers (see figure 1). The plane of the image is parallel to the scan pattern of 
the transducer. C-scan presentations are produced with an automated data acquisition system, such as a computer controlled immersion scanning system.

The C-scan method is based on the transmission of a very-high-frequency signal (up to $50 \mathrm{MHz}$ ) directed to the sample by the transducer. The sample and the transducer are submerged in a coupling medium (water in our case). The initial signal is partially reflected back to the transducer by the interface's grains, defects and porosities, or by other substantial differences in acoustic impedance in the sample and the signal of the transducer. If not fully reflected, the signal continues through the sample. In other words, between the initial pulse and the back-wall peaks there is an additional peak caused by the sound wave going from the water into the test material. This additional peak is called the "front wall peak". The ultrasonic tester can be adjusted to ignore the initial-pulse peak, so the first peak it will show will be the front-wall peak.

Some energy is lost when the ultrasound waves hit the test material, so the front-wall peak is slightly lower than the peak of the initial pulse. In return, the peak amplitudes and the time-of-flight of each returning signal are stored in a computer data file and processed offline to produce maps of the scanned area for the sample placed at a particular depth.

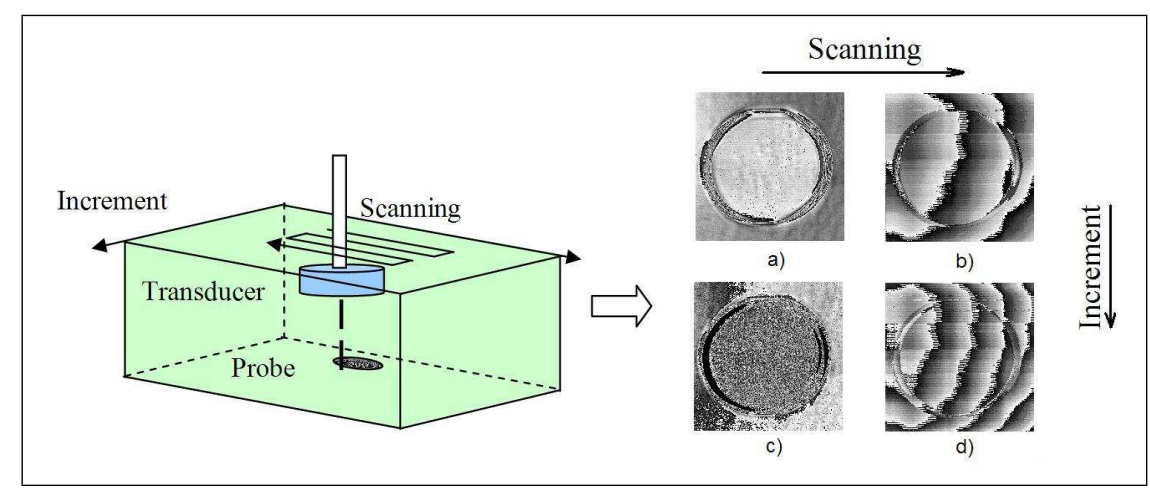

Figure 1: C-scan principle and samples of scanning

Figure 1 shows four ultrasonic C-scan images of a silicon solar plate (solar cell). All images were produced using a pulse-echo technique with the transducer scanning from above the sample in an immersion scanning system. For the C-scan image in figures 1.a and 1.c, the gate was set up to capture the amplitude of the sound reflecting from the head surface of the silicon plate. Light areas in the images indicate the regions that reflected a greater amount of energy back to the transducer. In the C-scan image in figures 1.b and 1.d, the gate was moved to record the intensity of the sound reflecting from the back surface of the plate. The details on the back surface are clearly visible, but the front surface features are also visible since the sound energy is affected by these features as it travels through the head surface of the silicon plate.

\subsection{Related work}

Because of the complexity of the real NDT setups, training of experts in nondestructive testing should take place in specially equipped laboratories. The cost of such a training configuration is rather great. This makes its implementation in academic laboratories difficult and, even so, the accessibility of students to the installation is reduced [16]. It also explains the small number of NDT training systems.

The Virtual Nondestructive Evaluation (NVDE) system proposed in [17] offers a full computerbased replica of a real NDT examination setup. Using NVDE, the user is able to generate the testing scenario, as trainer, to practice with the virtual setup and to perform assessment sessions to determine the performance level reached by the trainees. 
The CIVA software developed by CEA permits the visualization, optimization, and prediction of the performances of several testing techniques. Great effort is made in order to optimize the computing time so that the 3D models that are tested can be used in parametric studies, despite the potentially complex configurations. Moreover, CIVA can simulate the ultrasound wave propagation and highlight the defects inside the 3D models [18].

\subsection{The virtual environment}

In order to solve the problem of time limitation and lack of accessibility for more than one user that the real configuration presents, a virtual implementation of the scanner was developed. All functionalities of the real NDT installation were made accessible through EngView's 3D-immersive simulation software (see figure 2.a). This feature allows any user to practice the scanning procedure without any repercussions in case of faulty maneuvers.

This method offers a superior overview and understanding of the device and its mechanism of functioning. More precisely, the user is able to change the viewpoint inside the simulated environment (front/back, left/right, and up/down) and to navigate inside the virtual scanning device for a better view-point. These features allow the user to visualize the surface of the virtual scanned object during the simulation. The user can also move the three crane-like components of the virtual scanning device to virtually scan the simulated 3D probe.

The EngView setup was used during the second semester of 2007 in training sessions by engineering and physics senior students, organized in eight groups, each containing 25 students.

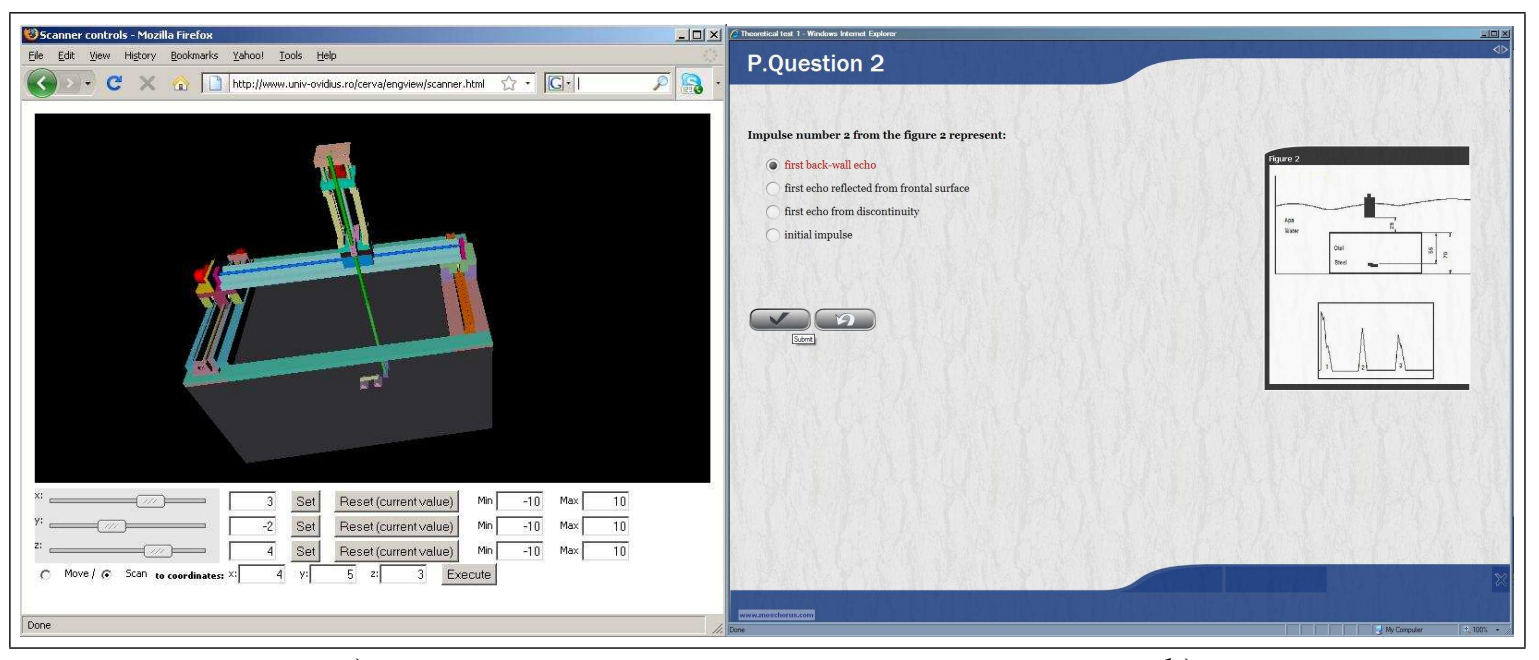

a)

b)

Figure 2: EngView : a) practical session, b) theoretical assessment session

The NDT curriculum requires one practical evaluation on the basis of six laboratory hours. As mentioned before, the NDT makes no exception in both theoretical and practical evaluation. To this end, the virtual environment contains pedagogical resources that provide users with access to theoretical background and evaluation as well as to practical sessions. Students can reproduce different types of realistic experiments using the EngView system by preparing the sample, changing the type of transducer, setting the parameters of the moving engines to establish the type of scanning procedure, and to make comparative studies. The students that work on the client machines in the EngView system are able to perform the same kind of analysis as in a real system.

The EngView system can be used either independently - not coupled to the real system - by installing it on a computer, or directly connected to the scanning device. The former option gives the advantage of 
supporting several students to train simultaneously using their home Internet-enabled computer. Through the latter option the device is actively controlled, serving as a safe and easy way to perform experiments when accurate data is required.

An assessment was organized on the basis of a multiple choice test containing ten pure theoretical and seven practical questions (see figure 2.b) to evaluate the knowledge acquired by the students. The time limit was 30 minutes to answer all questions. The practical evaluation had three steps: the experiment setup/calibration; the experiment itself; and the interpretation of the results. In the real configuration, about 30 minutes are necessary for the experiment per student, without any error recovery, so there is no possibility to try it twice during the exam. In this situation, it often occurs that the student uses the real NDT setup for the first time.

\subsection{Emulaction - a platform for distributing learning/training activities}

Although the solution described above provides the users with a more efficient learning environment, it does not support more users working together at the same time. To overcome this deficiency, we developed a context in which the simulations can take place.

This context is constituted by a virtual classroom that holds the fully functional 3D representations of each element from the real educational scenario. Organizing the learners in teams in the context offered by the "virtual classroom" metaphor helps to reduce most of the discrepancies between the individual knowledge levels increases communication and competition (in this order).

Competition becomes cooperation and aids the level of motivation. Hence, the complexity that may arise even in the most "simple" subjects is a non-declared motivational factor when introduced gradually. If the students' needs are satisfied and their expectations are met, they will strive to develop their professional competences. Indirectly, students contribute to the development of the learning context (see figure $3)$.

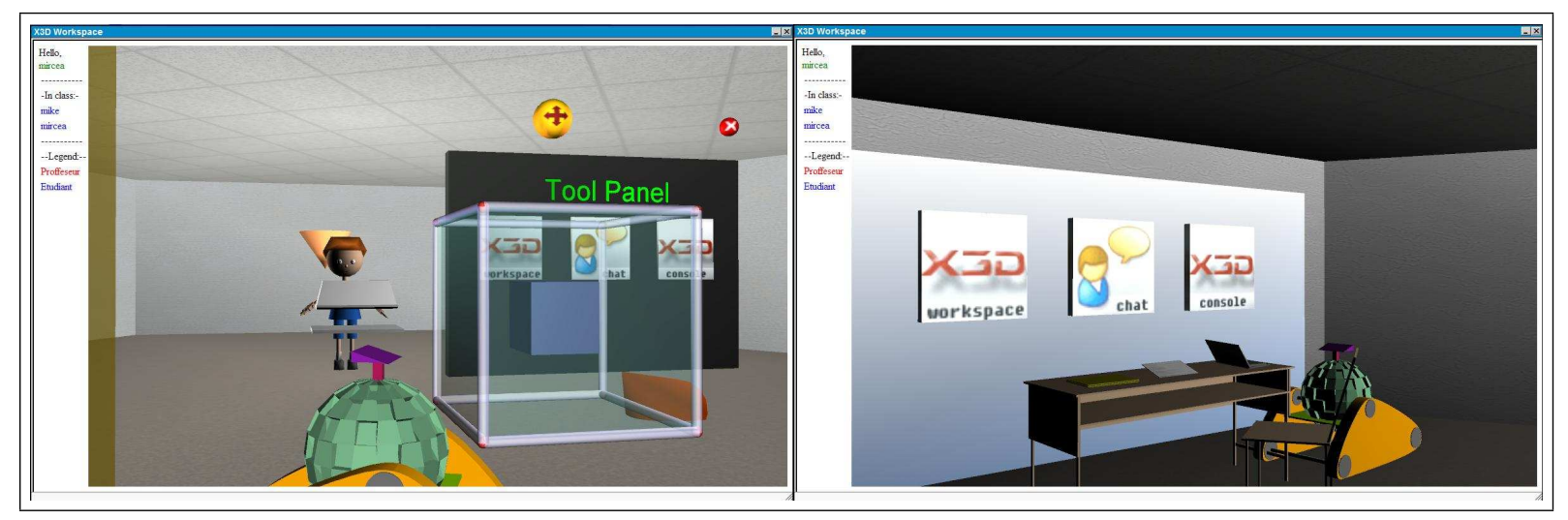

a)

b)

Figure 3: Shared training environment (EMULACTION project): a) Users sharing a task, b) Virtual office containing theoretical material and assesment tools

Because the students share the real environment, we want them to share a similar virtual environment also. Students naturally start to form small work teams in the virtual setup, based on the real environment configuration. Later, these teams may evolve based on the complementary knowledge that the team members possess, in order to assure a higher level of team performance.

Shared experiences provide different, perhaps even complementary perspectives to the lesson, depending on each individual.

Combining specific tasks in a distributed platform enables the users to collaborate and focus on the same target, share knowledge and impressions. Working in teams can bring great advantages to the 
learning experience, as users can communicate and coordinate each other's actions in real time, while conducting the experiments.

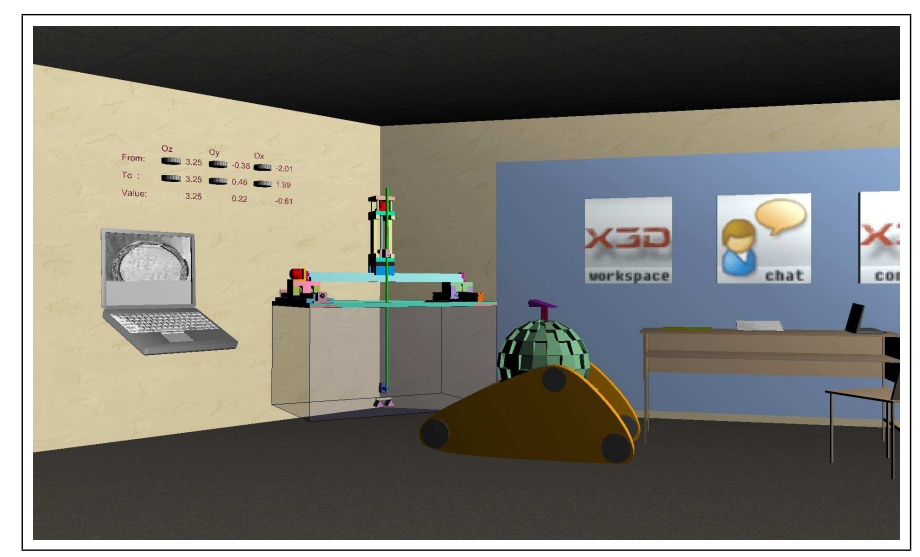

Figure 4: EngView-based shared training session

Figure 4 shows an example of such a context, where the students that have passed the theoretical assessment have access to the virtual replica of the NDT scanning installation. Here they may test different scanning parameters and different probe materials while visualizing the same EngView environment. A virtual laptop gradually displays the scanned probe and can receive commands to either start or stop the scan. The scanning device is a fully functional replica of the real equipment, and the cranes from the standard version of EngView have been replaced by virtual disks that can be rotated to achieve the desired position of the start and end positions. The visual feedback is coherent with the used scanning parameters and may give hints to the trainee concerning the task currently in progress.

\section{Implementation-related aspects}

Our educational virtual environments are currently based on the assumption that knowledge and skills acquired in a VR-based environment will be transferred to the real world. The effectiveness of such an environment depends on the user's capability to apply the knowledge and/or the skills acquired to its real world counterpart.

The current learning materials are implemented using the Moodle [19] platform for the text and multimedia resources (DOC, PDF, PPT, AVI, or JPEG files) as well as 3D virtual environments.

Concerning textual and multimedia support, we explored the Moodle facilities to align the pedagogical context with the Sharable Content Object Reference Model norms [20]. Moreover, we manage the users' access to the corresponding course materials according to their curricula and the course materials. Therefore, the tutors have the ability to create, modify, and publish educational materials, such as courses, seminars, homeworks, project subjects, tests, and so on. Furthermore, using such a system, the administrator is able to manage the courses, the users, the groups of students, and the students enrolling in each course.

Our 3D environments are developed using VRML [21] and/or ARéVi API [22]. The ARéVi API is open-source, $\mathrm{C}++$ and OpenGL based, and adaptive to different configurations, ranging from desktops to 3D stereoscopic immersion systems. To put all together, we use a reactive agent-based architecture [23]. This architecture assures the user's immersion and evolution within the virtual space.

To ensure the distributed activities, we have adopted the Linux, Apache [24], MySQL [25], and PHP [26] based solution. Because our educational environment is mostly 3D-oriented, we chose to build it based on the AJAX/AJAX3D technology [27, 28] and X3D/VRML language [29, 21]. AJAX provides optimal update speed between the client and the server by simulating a direct connection, while X3D 
has the advantage of having an accessible structure that can be controlled with the JavaScript engine through a browser plug-in called FluxPlayer [30]. FluxPlayer is easily installable on Windows (XP and Vista) operating systems for Firefox [31] and Internet Explorer browsers. The scene access interface (SAI) is achievable also through Java, but in this case we considered that having an applet to control the environment was unnecessary. This approach is still at the beginning as more and more gamelike browser-based applications spread over the internet. This launch is facilitated by the increase in processing power of the personal computers, and by the ever-evolving internet browsers that are able to faster process web content. To this end, this architecture can be considered modern and unique in the context of educational purposes.

PHP and MySQL are in charge of the user account and database management. The system currently supports two account types: student and teacher, each enabling users to perform certain actions depending on their status. Apart from proving a high level of performance, the system is easy to install on any operating system that supports PHP and MySQL. Although the update speed is not real-time due to the impossibility of establishing a direct connection with the server through this architecture, this was not a factor of decision because the main objective of this application is only to provide users with a functional collaborative environment in which they can practice.

Assuming a high number of users connected to the system simultaneously, the application was optimized to cache new events to prevent unnecessary communication with the server that would cause it to slow down. Updates are transmitted using the XML format for better information structuring. XML not only brings ease in the use of the received data, but also makes the system adaptable to changes brought to its structure. Each client of the application requests updates from the server at customizable time intervals, depending on the connection speed.

The virtual classrooms also offer users tools to communicate with each other and submit results for verification, after experiments have been completed. Among these tools are the button toolbar, books and files, which are also viewable by all participants when activated (see figures 3 and 4).

The environment is designed to be customizable by the teachers that want to hold a course in of different curricula. The teacher account type offers users the freedom to create a personalized classroom, suitable for the course that needs to be held, containing adequate tools and devices for the students to use. This way, the customized classroom is dynamically generated by the application, and becomes ready for the students to join.

\section{Discussions}

In order to evaluate the efficiency of the virtual setup, we gathered completion time information from students trained in the classical manner and those who benefited from the virtual practice (see figure 5).

We observed that using only the traditional training sessions is neither motivating nor time-efficient. The probability of failure because of poor practical skills and/or errors that may appear during the experiment is too high for the current curricula. On the other hand, by offering students the possibility of practicing in the virtual configuration before the real one, they became more confident in their own potential due to the chance to recover from errors and to experiment with more training situations.

In addition, the number of hours dedicated by the faculty's regulations for training and practice with the scanning device is considerably small. EngView makes seminars less expensive by using complex immersive and interactive simulations which are accessible over the internet. Moreover, it brings students closer to the practical part of their education and helps them better comprehend each learned concept.

In order to evaluate the system's impact on the user's learning/training process we have compared the assessment results obtained in classical training context with those obtained after shared 3D-setup was used (see figures 6 and 7). Table 1 contains the repartition of the users' results.

For each answer data set we determined the characteristic values as the average, the mode and the 


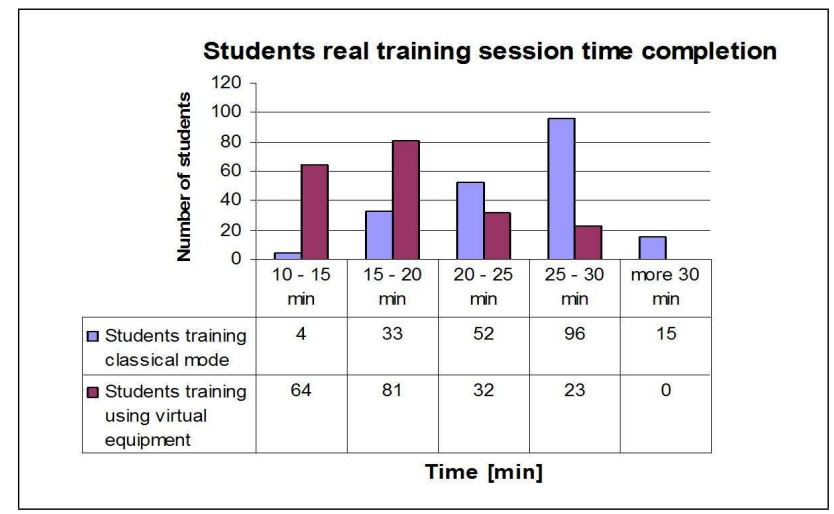

Figure 5: Comparison of training completion time in real configuration with and without virtual training sessions, respectively

variance. The first two values represent the central tendency while the variance represents the dispersion degree around the mean. The mode is the most frequent value that appears in the data set.

\section{Classic assesment 3D assesment}

\begin{tabular}{|c|c|c|c|c|}
\hline \hline $\begin{array}{c}\text { Possible } \\
\text { values }\end{array}$ & $\begin{array}{c}\text { Theoretical } \\
\text { questions }\end{array}$ & $\begin{array}{c}\text { Practical } \\
\text { questions }\end{array}$ & $\begin{array}{c}\text { Theoretical } \\
\text { questions }\end{array}$ & $\begin{array}{c}\text { Practical } \\
\text { questions }\end{array}$ \\
\hline 1 & 2 & 8 & 0 & 2 \\
2 & 7 & 7 & 0 & 3 \\
3 & 10 & 19 & 10 & 12 \\
4 & 17 & 37 & 16 & 23 \\
5 & 13 & 46 & 8 & 48 \\
6 & 18 & 45 & 22 & 40 \\
7 & 29 & 38 & 33 & 72 \\
8 & 43 & 0 & 41 & 0 \\
9 & 39 & 0 & 47 & 0 \\
10 & 22 & 0 & 23 & 0 \\
\hline Total & 200 & 200 & 200 & 200 \\
Mean & 7.025 & 4.965 & 7.39 & 5.6 \\
Variance & 5.134375 & 2.503775 & 3.8179 & 1.93 \\
Mode & 8 & 5 & 9 & 7 \\
\hline \hline
\end{tabular}

Table 1: User results in both classical and 3D training contexts and characteristic values

Based on these values and the corresponding charts (see figures 6 and 7) we can conclude that the differences indicate a significant overall improvement in the case of using the 3D setup.

In order to verify that the improvement brought by the 3D setup is indeed significant, we also applied a statistical $\mathrm{T}$ test for mean comparison between the two samples assuming unequal variances. The resulting P-values corresponding with theoretical assessments and practical assessments, i.e. 0.043032 and 0.000014 respectively, are smaller than 0.05 ; hence the difference between the means of the 2 samples is significant.

In other words, since the mean of the 3D setup is obviously higher than the classical approach, we conclude that the 3D method brings significant improvement in the training process.

We have also implemented an anonymous questionnaire that focuses on both the EngView's user interface and the environment content. We have opted for a "five-level-choice" questionnaire, with the following grades: "very poor", "poor", "acceptable", "good", and "excellent". 


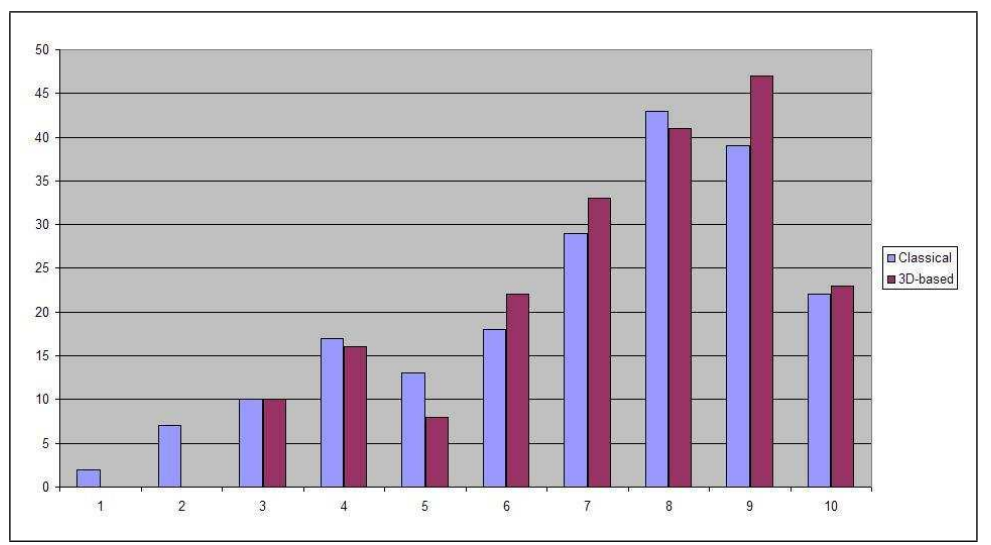

Figure 6: Results of the theoretical assessment using classical approach v.s. 3D-based one

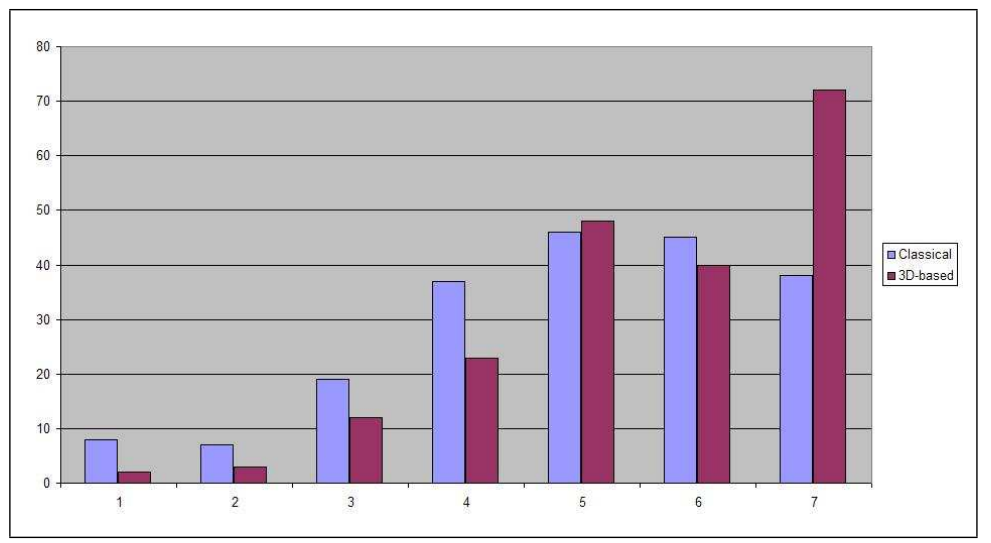

Figure 7: Results of the practical assessment using classical approach v.s. 3D-based one

\begin{tabular}{lccccc}
\hline \hline Question / Answer & very poor & poor & acceptable & good & excelent \\
\hline \hline The interface is self-explanatory? & $0 \%$ & $0 \%$ & $16.2 \%$ & $83.5 \%$ & $0.3 \%$ \\
\hline $\begin{array}{l}\text { The environment helps me to } \\
\text { identify the key concept? }\end{array}$ & $0 \%$ & $0.28 \%$ & $15.6 \%$ & $83.1 \%$ & $0.02 \%$ \\
\hline $\begin{array}{l}\text { How natural was the interaction } \\
\text { with existing objects? }\end{array}$ & $0 \%$ & $0.17 \%$ & $73.2 \%$ & $12.97 \%$ & $13.66 \%$ \\
\hline $\begin{array}{l}\text { How did you find the virtual NDT } \\
\text { setup feedback? }\end{array}$ & $0 \%$ & $0 \%$ & $1.3 \%$ & $97.2 \%$ & $1.5 \%$ \\
\hline $\begin{array}{l}\text { The reuse of the capabilities in } \\
\text { real setup that where obtained } \\
\text { in virtual setup }\end{array}$ & $0 \%$ & $0 \%$ & $3 \%$ & $79 \%$ & $18 \%$ \\
\hline \hline
\end{tabular}

Table 2: Engview evaluation questionary 
As the results show (table 2), despite the specificity of the EngView environment, what we want to convey to all users of our virtual environments is self-confidence and team-oriented contexts. The virtual space has to motivate users to study the environment by direct and constructive observation of its components, without any temporal or geographical constraints. By simulating real setups into virtual spaces we encourage the users to be active situated actors in self-explanatory pedagogical contexts.

\section{Conclusions and future work}

As previously stated at the beginning of this contribution, the subtle goal of this work is to prove whether virtual 3D environments are able to increase the efficiency and of learning processes and their capacity of being evolutionary. First of all, people need feedback in order to comprehend the activities they perform; the lack of feedback is a major issue when dealing with the educational context because materials and equipment are often too expensive to purchase in large amounts. Having virtual simulations of the real training material lowers the costs of training, and increases the number of students that are able to be trained using them. Secondly, it was proved that collaboration increases the quality of learning, but not all virtual environments support multiple user access. Using a distributed platform that can implement various live training sessions makes possible the evolution of teams of students while training. Users receive feedback from their own actions as well as from other's, this way maximizing the intake of information. Thirdly, statistics based on the users' responses show that learning speed is greatly increased when using virtual environments in addition to classical methods. To this end, the point in adopting interactive 3D worlds in the educational context has been proven.

One of the central directions of our efforts is to use ontologies in content management and deployment. This may be useful in producing similar pedagogical situations that use different content. This may also allow us to introduce agent-oriented tutors that can evaluate the users' actions inside the 3D space.

\section{Acknowledgements}

Our contribution is an extended version of the paper [32] previously published in the Proceedings of the 3rd International Conference on Virtual Learning (ICVL'08). This work is partially funded by the following projects: INTUITION (FP6-IST-NMP-1-507248-2), EMULACTION (Fonds Francophones des Inforoutes - ref.no. 14G023), and TOMIS (PN II: 11-041/2007, National Centre of Programs Management).

\section{Bibliography}

[1] P. Milgram, H. Takemura, A. Utsumi, F. Kishino, Augmented Reality: A class of displays on the reality-virtuality continuum, SPIE Vol. 2351, Telemanipulator and Telepresence Technology, pp. 282-292, 1994.

[2] G. Burdea, Ph. Coiffet, Virtual Reality Technology Second Edition with CD-ROM, Wiley, New Jersey, 2003, ISBN 0471360899.

[3] D.Zelter, Autonomy, interaction and presence, Presence, Vol. 1(1), pp. 127-132, 1990.

[4] A. Wilson, The promise of Situated Cognition, In An Update On Adult Learning Theory, Ed. By S.B.Merriam, pp. 71-79, San Francisco, Jossey-Bass, 1993.

[5] J. Lave, E. Wenger, Situated learning. Legitimate peripheral participation, Cambridge, University of Cambridge Press, 1991. 
[6] G. Taxen, A. Naeve, CyberMath: A Shared Virtual Environment for Mathematics Exploration, Center for User Oriented IT Design, Royal Institute of Technology, Technical Report CID-129, Stockholm, Sweden, 2001.

[7] B. Loftin, M. Engelberg, R. Benedetti, Applying Virtual Reality in Education: A prototypical Virtual Physics Laboratory, In Proceedings of IEEE Symposium on Research Frontiers in Virtual Reality, San Jose, CA, pp. 67-74, 1993.

[8] J.P. Gerval, Y. Le Ru, VELab: A Virtual Lab for Electronics Virtual Experiments, Advanced Technology for Learning, Volume 3 - Issue 2, ACTA Press, 2006.

[9] N. Doukas, A. Andreatos, Advancing Electronic Assessment, International Journal of Computers, Communications \& Control, Vol.II, No.1, pp. 56-65, 2007.

[10] W. L. Johnson, J. Rickel, Steve: An Animated Pedagogical Agent for Procedural Training in Virtual Environments, Sigart Bulletin, ACM Press, vol. 8, number 1-4, pp. 16-12, 1997.

[11] J.-M. Burkhardt, D. Lourdeaux, D. Mellet-d'Huart: La conception des environnements virtuels d'apprentissage (the design of training virtual environments). In: P. Fuchs \& G. Moreau (eds.): Le traité de la réalité virtuelle. Paris, Presses de l'Ecole des Mines, 2003.

[12] F.G. Hamza-Lup, I. Sopin, Haptics and Extensible 3D in Web-Based Environments for e-Learning and Simulation, 4th International Conference on Web Information Systems and Technologies (WEBIST), May 4-7, Funchal, Madeira, Portugal, 2008.

[13] C. Roloson, J. Zirnhelt, PERFORMANCE BASED QUALIFICATION: AN NDT SERVICE INDUSTRY PERSPECTIVE, paper No. 744, CD-ROM Proceedings of the 16th WCNDT 2004 - World Conference on NDT, Aug 30 - Sep 3, Montreal, Canada, http://www.ndt.net/abstract/wcndt2004/744.htm, 2004.

[14] J. Bruner, Actual Minds, Possible Worlds, Harvard University Press, Cambridge, MA., 1986.

[15] CERVA, http://www.univ-ovidius.ro/cerva/engview, 2007.

[16] P.N. Shkatov, D.V. Bydanov, S.V. Kluev, Simulator for Education of Eddy Current Testing Specialists, Proc. of The 17th World Conference on Nondestructive Testing, 25-28 Oct 2008, Shanghai, China.

[17] D.H. Harris, J. Spanner, Virtual NDE operator training and qualification, NDT.net - October 1999, Vol. 4 No. 10.

[18] S. Lonné, L. de Roumilly, L. Le Ber, S. Mahaut, G. Cattiaux , Experimental Validation of CIVA Ultrasonic Simulations, International Conference on NDE in relation to structural Integrity for Nuclear and Pressurised Components, 2006.

[19] Moodle, http://moodle.org, Feb., 2009.

[20] SCORM, Sharable Content Object Reference Model, http://www.adlnet.gov/scorm/, Feb., 2009.

[21] VRML, Virtual Reality Modeling Language, http://www.web3d.org/x3d/specifications/vrml/, Feb., 2009.

[22] P. Reignier, F. Harrouet, S. Morvan, J. Tisseau, T. Duval, ARéVi: A Virtual Reality Multiagent Platform, Lectures Notes in Computer Science, Volume 1434, ISSN: 0302-9743, http://www.cerv.fr/fr/activites/AReVi.php, 1998. 
[23] D.M. Popovici, Modeling the space in virtual universes, PhD Thesis, Politehnica University of Bucharest, 2004.

[24] Apache, http://www.apache.org, Feb., 2009.

[25] MySQL, http://www.mysql.com, Feb., 2009.

[26] PHP, http://www.php.net, Feb., 2009.

[27] AJAX, http://www.ajax.org, Feb., 2009.

[28] AJAX3D, http://www.ajax3d.org/, Feb., 2009.

[29] X3D, http://www.web3d.org, Feb., 2009.

[30] FluxPlayer, http://sourceforge.net/projects/flux, Feb., 2009.

[31] Firefox, http://www.firefox.com, Feb., 2009.

[32] D.M. Popovici, J.P. Gerval, F.G. Hamza-Lup,N. Popovici,M. Polceanu,R. Zagan: Learning Distributed Activities Inside 3D Virtual Spaces, Proceedings of The $3^{\text {rd }}$ International Conference on Virtual Learning, pp. 289-296, Constanta, 2008.

Dorin-Mircea Popovici (08/07/1968) obtained his PhD in Computer Science at Politehnica University of Bucharest, Romania (2004). He actively participates in several international and national projects, all oriented on the use of Virtual Reality in human activities like learning, education and culture heritage. He had written the book "An insight of 3D virtual environments" in romanian, published by Ed. Muntenia, Constanta (2007). He is the founder and the leader of The Research Team in Virtual and Augmented Reality of the OVIDIUS University of Constanta (CERVA). (For more information please see http://www.univ-ovidius.ro/cerva)

Jean Pierre Gerval (12/12/1957) obtained his $\mathrm{PhD}$ in automation from the University of Valenceinnes in France (1987). He has been project manager at the "Institut d'Informatique Industrielle" in Brest, France (1988-2003) and associate professor of computer sciences at the "Ecole Nationale d'Ingénieurs" de Brest (1993-2003). He is currently the head of the Computer Science Department at the "Institut Supérieur de l'Electronique et du Numérique" in Brest. He received the "Best Software System" Award from IASTED International Conference on Computers and Advanced Technology in Education - Oranjestad - Aruba - August 2005 for the development of the Virtual Lab for Electronics. His research interests include distributed virtual reality and virtual environments especially dedicated to pedagogical applications.

Dr. Hamza-Lup (20/03/1976) received a B.Sc. in Computer Science from Technical University of Cluj-Napoca, Romania, the MS. and Ph.D. in Computer Science from University of Central Florida, Orlando in 2001 respectively 2004. He is involved with research and development of medical simulation systems and medical training tools that use 3D imaging components and virtual reality paradigms. His research interests are: Human Computer Interaction, Distributed Systems, Virtual Reality and Simulation/Training for Medical Procedures. He received grants from several organizations including NASA, MD Anderson Cancer Center (Orlando) and has served as co-PI on several STTR and SBIR grants. In 2003 he has received the Link Foundation Fellowship in Advanced Simulation and Training and in 2005 the Distinguished Service Award for mentoring students in science. (For more information please see http://www.cs.armstrong.edu/felix)

Ronan Querrec (18/09/1973) had his Phd in 2001 in the science university of Brest. He is professor assistant in Computer Science and works at the CERV. His reasearch work is about virtual environment for training. In this theme, he works on the MASCARET project, a virtual environment metamodel. 
Mihai Polceanu (22/12/1988) is currently a student in Computer Science at the OVIDIUS University of Constanta. As member of the CERVA team, he works in the EMULACTION project as architecture designer and programmer. His domains of interests are: Programming, Virtual Reality, Web Technologies, Cryptography, Cryptanalysis and Data Security.

Norina Popovici (29/04/1970) obtained her PhD in economics (2005). Her domains of interest are management, human resources management, project management, and usability of web technologies in management and educational systems.

Remus Zăgan (11/06/1967) had his PhD in Industrial Engineering at Technical University "Gh.Asachi", Ia si in the field of vibration, ultrasound, material characterization, modeling and simulation, He is currently the Dean of Mechanical Industrial and Maritime Engineering Faculty, "Ovidius" University of Constanta. He had managed 7 national grants, 1 international grant and deposed 1 patent. He has several books in wavelets analysis of noise and vibrations, modeling and simulations of production systems. 